\title{
Effect of extraction treatment on upper airway dimensions in patients with bimaxillary skeletal protrusion relative to their vertical skeletal pattern
}

\author{
$\mathrm{Ha}-\mathrm{Nul} \mathrm{Cho}{ }^{\mathrm{a}}$ (1) \\ Hyun Joo Yoon ${ }^{\mathrm{a}}$ \\ Jae Hyun Park ${ }^{\mathrm{a}, \mathrm{b}}$ \\ Young-Guk Park ${ }^{\mathrm{c}}$ \\ Su-Jung Kim ${ }^{\mathrm{c}}$ (1)
}

${ }^{a}$ Department of Dentistry, Graduate School, Kyung Hee University, Seoul, Korea

${ }^{\mathrm{b}}$ Postgraduate Orthodontic Program, Arizona School of Dentistry \& Oral Health, A.T. Still University, Mesa, AZ, USA

'Department of Orthodontics, School of Dentistry, Kyung Hee University, Seoul, Korea

\begin{abstract}
Objective: To investigate dimensional changes in regional pharyngeal airway spaces after premolar extraction in bimaxillary skeletal protrusion (BSP) patients according to vertical skeletal pattern, and to further identify dentoskeletal risk factors to predict posttreatment pharyngeal changes. Methods: Fiftyfive adults showing BSP treated with microimplant anchorage after four premolar extractions were included in this retrospective study. The subjects were divided into two groups according to the mandibular plane steepness: hyperdivergent (Frankfort horizontal plane to mandibular plane [FH-MP] $\geq 30$ ) and nonhyperdivergent groups ( $\mathrm{FH}-\mathrm{MP}<30)$. The control group consisted of 20 untreated adults with skeletal Class 1 normodivergent pattern and favorable profile. Treatment changes in cephalometric variables were evaluated and compared. The association between posttreatment changes in the dentoskeletal and upper airway variables were analyzed using linear regression analysis. Results: The BSP patients showed no significant decrease in the pharyngeal dimensions to the lower level in comparison with controls, except for middle airway space (MAS, $p<0.01$ ). The upper airway variable representing greater decrease in the hyperdivergent group than in the nonhyperdivergent group was the MAS $(p<0.01)$. Posttreatment changes in FH-MP had negative correlation with changes in MAS $(\beta=-0.42, p<0.01)$ and inferior airway space $(\beta=$ $-0.52, p<0.01)$ as a result of multivariable regression analysis adjusted for sagittal skeletal relationship. Conclusions: Decreased pharyngeal dimensions after treatment in BSP patients showed no significant difference from the normal range of pharyngeal dimensions. However, the glossopharyngeal airway space may be susceptible to treatment when vertical dimension increased in hyperdivergent BSP patients.
\end{abstract}

[Korean J Orthod 2021;51(3):166-178]

Key words: Airway, Cephalometrics, Bimaxillary skeletal protrusion, Vertical skeletal pattern

Received June 3, 2020; Revised November 11, 2020; Accepted December 9, 2020.

Corresponding author: Su-Jung Kim.

Professor and Department Chair, Department of Orthodontics, School of Dentistry, Kyung Hee University, 26 Kyungheedae-ro, Dongdaemun-gu, Seoul 02447, Korea.

Tel+82-2-958-9390 e-mail ksj113@khu.ac.kr

How to cite this article: Cho HN, Yoon HJ, Park JH, Park YG, Kim SJ. Effect of extraction treatment on upper airway dimensions in patients with bimaxillary skeletal protrusion relative to their vertical skeletal pattern. Korean J Orthod 2021;51:166-178.

(C) 2021 The Korean Association of Orthodontists.

This is an Open Access article distributed under the terms of the Creative Commons Attribution Non-Commercial License (http://creativecommons.org/licenses/by-nc/4.0) which permits unrestricted non-commercial use, distribution, and reproduction in any medium, provided the original work is properly cited. 


\section{INTRODUCTION}

Bimaxillary protrusion (BMP) is defined as a condition wherein the upper and lower incisors are protrusive or proclined, leading to lip protrusion, which mostly corresponds to bimaxillary dentoalveolar protrusion. ${ }^{1}$ In a specific sense, bimaxillary skeletal protrusion (BSP) is characterized by protrusive upper and lower jaws with or without incisor proclination. ${ }^{2}$ Most patients with skeletal and/or dentoalveolar protrusion are treated with microimplant anchorage for maximum anterior retraction after extraction of four premolars with the aim of esthetic improvement. ${ }^{3-5}$ In particular, BSP patients require greater retrusion of both A-point and B-point areas concomitant with incisor retraction than BMP patients. However, there may be concerns regarding the effects of a decreased oral cavity with a retruded anterior alveolus and subsequent parapharyngeal tissue displacement on the airway dimensions and respiratory function. ${ }^{6,7}$ On the other hand, the pharyngeal airway dimensions of BSP patients are presumed to be initially large enough to maintain normal airway patency even after extraction treatment, albeit with no scientific evidence. ${ }^{8}$

Previous studies have investigated the pharyngeal dimensional changes after premolar extraction treatment in BMP patients. A few studies found significant constriction of the velopharyngeal space behind the soft palate, glossopharyngeal space behind the tongue base, and/or hypopharyngeal space below the epiglottis base after extraction treatment, ${ }^{9,10}$ whereas one study reported no significant posttreatment changes at any level of the pharyngeal spaces." These contradictory findings might be due to the differences in sample characteristics between a skeletal Class 1 BMP with a well-developed chin and skeletal Class II BMP with a retruded chin. These two patterns tend to show different initial pharyngeal morphologies and dimensions, which may respond differently to the dentoskeletal treatment changes. ${ }^{12}$ Moreover, no previous study has differentiated the BSP from BMP, which requires a different amount and pattern of anterior retraction of teeth and apical bone, to demonstrate the posttreatment pharyngeal airway changes.

Notably, the vertical skeletal pattern may affect the treatment outcome of the pharyngeal dimension in BSP patients, because a hyperdivergent vertical pattern with steep mandibular and occlusal planes is known to be a predisposing factor for pharyngeal narrowing and collapsibility. ${ }^{13-15}$ One unique study reported no significant difference in the pharyngeal changes between hyperdivergent and nonhyperdivergent groups in BMP patients treated with premolar extraction. ${ }^{10}$ More importantly, an increase in the vertical dimension by clockwise mandibular rotation after treatment may contribute to pharyngeal narrowing; however, the effects of vertical skeletal changes on the pharyngeal dimensions in BSP patients has not been discussed so far.

Existing evidence does not support the notion that extraction treatment may induce a detrimental effect on respiratory function or obstructive sleep apnea (OSA). ${ }^{16}$ Although pharyngeal narrowing may be noted after treatment in two-dimensional or three-dimensional radiographic images, most patients show the potential for functional adaptation to normal function. ${ }^{17,18}$ However, some orthodontic patients may have risks or symptoms of OSA in relation to the hidden neuromuscular pathophysiology, which might increase the susceptibility to the pharyngeal change after orthodontic treatment. ${ }^{19}$ Therefore, orthodontists should reflect the possible pharyngeal responses to various types of orthodontic treatments in daily practice. It is worth questioning whether the posttreatment pharyngeal airway dimensions would be stable in BSP patients despite the greater retraction of the anterior teeth and alveolar bone due to their initially larger patency than that in the normal skeletal pattern, or it might be unfavorably changed by some dentoskeletal risk factors. The objective of this retrospective study was to investigate the dimensional changes in regional pharyngeal airway spaces after premolar extraction treatment in BSP patients according to their vertical skeletal pattern. Further, any dentoskeletal risk factors that might be used to predict posttreatment pharyngeal changes in BSP patients were also evaluated.

\section{MATERIALS AND METHODS}

\section{Subjects}

Seventy-two adult patients who were diagnosed with BSP and treated at the Department of Orthodontics, Kyung Hee University Dental Hospital were identified. Fifty-five patients ( 10 males and 45 females) with a mean age of $23.4 \pm 5.21$ years (range, 18.2 to 34.9 years) who met the following inclusion criteria were included: (1) maxillary and mandibular protrusion as indicated by A-point and B-point protrusion, respectively (Nper-A $\geq 2 \mathrm{~mm}$, Nper-B $\geq-3 \mathrm{~mm}$ ); (2) arch length discrepancy $\leq 4 \mathrm{~mm}$; (3) extraction of four first premolars; (4) microimplant anchorage for maximum anterior retraction; (5) pre- and posttreatment lateral cephalograms taken in a natural head position, and in a breathholding position after the end of expiration to obtain reproducible pharyngeal caliber ${ }^{20}$; and (6) no evidence of adenoid and/or tonsillar hypertrophy in the cephalogram. The exclusion criteria were as follows: (1) chronic medication; (2) history of pharyngeal soft tissue surgery; (3) obesity with body mass index over $25 \mathrm{~kg} / \mathrm{m}^{2}$; and (4) bidentoalveolar protrusion with no skeletal protrusion. This study was approved by the Institutional Review Board of Kyung Hee University Dental Hospital (IRB No. 

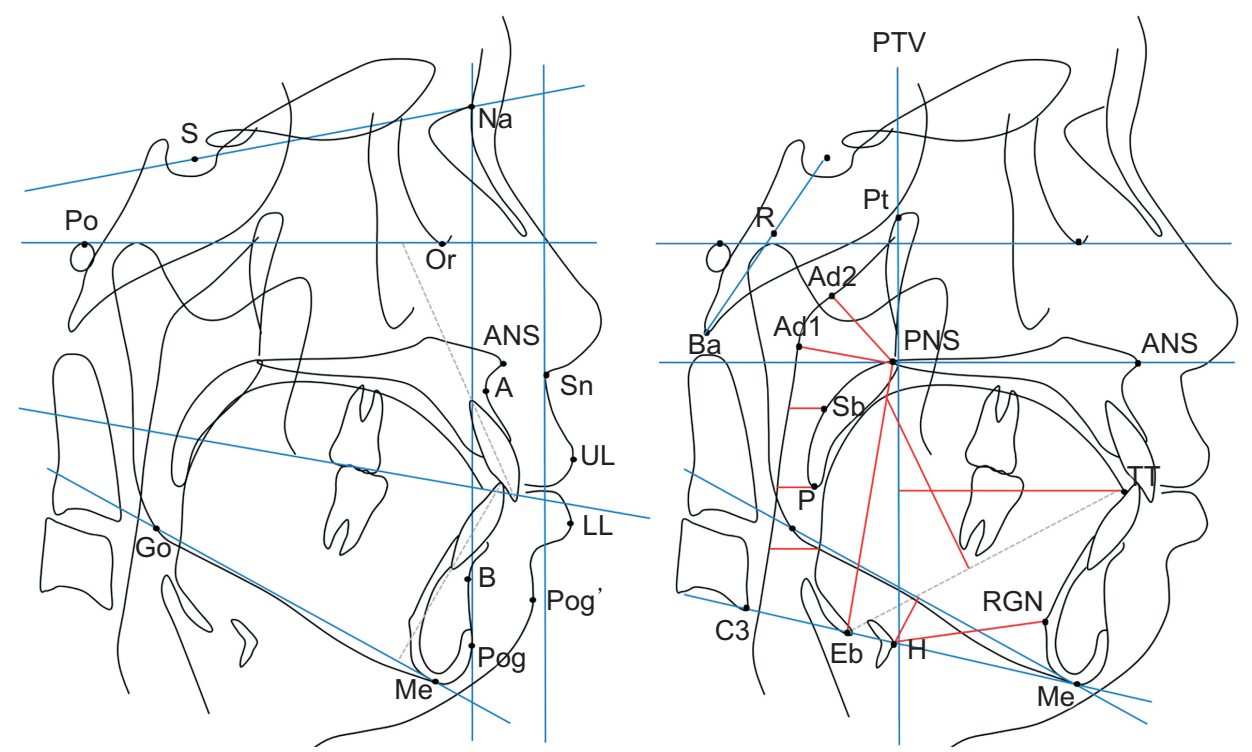

Figure 1. Dentoskeletal (A) and pharyngeal (B) cephalometric landmarks and measurements.

$S$, sella; Na, nasion; Po, porion; Or, orbitale; $A N S$, anterior nasal spine; PNS, posterior nasal spine; $A$, point $A$; $B$, point $B$; Pog, pogonion; Me, menton; Go, gonion; Sn, subnasale; UL, upper lip; LL, lower lip; Pog'; soft-tissue pogonion; Pt, pterygomaxillary point; $A d 1$, adenoid point 1 ; $A d 2$, adenoid point 2; Ba, basion; $\Pi$, tongue tip; $H$, hyoid bone; RGN, retrognathion; C3, third vertebra; $P$, tip of soft palate; $R$, midpoint of S-Ba line; Sb, belly of soft palate; Eb, base of epiglottis.

See footnote of Table 1 for the definition of measurements.

\section{KH-DT19015).}

The subjects with BSP were divided into two groups according to the initial mandibular plane angle (FHMP): the hyperdivergent group $(\mathrm{n}=27)$ with an FH-MP of $30^{\circ}$ or more; and the nonhyperdivergent group $(\mathrm{n}=$ 28) with FH-MP less than $30^{\circ}$. A control group was set up to basically evaluate whether the pretreatment and posttreatment pharyngeal dimensions in the BSP group showed significant differences, respectively. The control group consisted of 20 untreated adults (8 males and 12 females; mean age, $25.1 \pm 4.8$ years) with skeletal Class 1 normovergent patterns and a favorable facial profile and incisor inclination. All subjects were treated using $0.022 \times 0.028$-inch (in) self-ligating brackets (Clippy-C; Tomy, Tokyo, Japan). Maximum anterior retraction was performed using sliding mechanics with $0.019 \times 0.025$-in stainless steel wires and microimplant anchorage on all quadrants.

Pretreatment (T0) and posttreatment (T1) lateral cephalograms were evaluated by one expert orthodontist at two-week intervals. Cephalometric measurements were consisted of 17 dentoskeletofacial and 14 upper airway variables (Figure 1). Dimensions of the pharyngeal airway were evaluated as follows (Figure 2): the nasopharyngeal space above the palatal plane was represented by PNS-Ad1 and PNS-Ad2; the velopharyngeal space behind the soft palate was represented by superior posterior airway space (SPAS); and the glossopharyngeal

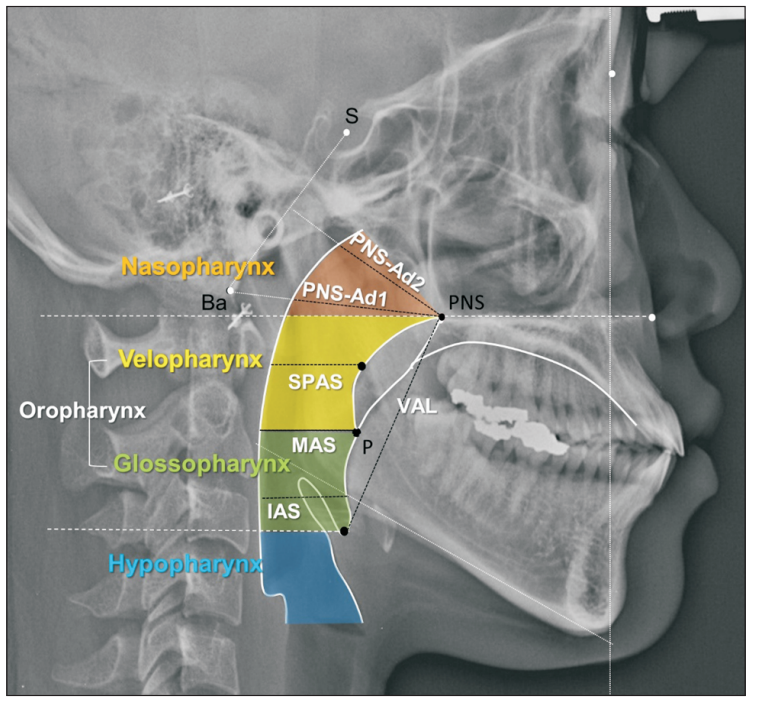

Figure 2. Cephalometric pharyngeal measurements to describe each pharyngeal airway section: PNS-Ad2 and PNS-Ad1 for the nasopharynx; superior posterior airway space (SPAS) for the velopharynx behind the soft palate; middle airway space (MAS) and inferior airway space (IAS) for the glossopharynx behind the tongue base; and vertical airway length (VAL) for the pharyngeal airway length."

See Figure 1 and footnote of Table 1 for the definition of other landmarks or measurements. 
space behind the tongue base was described as middle airway space (MAS) and inferior airway space (IAS).

\section{Statistical analysis}

To assess the error in cephalometric analysis, measurement sets were obtained twice with a two-week interval and analyzed by a paired $t$-test. The mean intra-observer differences were less than $0.45 \mathrm{~mm}$ and $0.64^{\circ}$ in linear and angular measurements, respectively. No significant differences were found in any of the variables. Pearson correlation coefficients for all variables represented a significant range of 0.93 to 0.97 , showing the reliability of the measurements.

A Shapiro-Wilk test confirmed the normal distribution of the data. Overall treatment change $(\Delta T)$ was analyzed using a paired $t$-test, and intergroup comparison was performed using an independent $t$-test. Univariable linear regression analyses were conducted to detect any correlation between posttreatment dentoskeletal changes and upper airway changes. Then, multivariable linear regression analysis was performed to determine whether the posttreatment changes in vertical skeletal divergency (FH-MP) affect the changes in upper airway variables when adjusted for the sagittal skeletal relationship (ANB angle). SPSS software, version 22.0 (IBM Corp., Armonk, NY, USA) was used, and all data were expressed as mean and standard deviation $(p<0.05)$.

\section{RESULTS}

Overall treatment changes in the BSP patients in comparison with control samples (Table 1)

Before treatment, BSP patients showed no significant differences in pharyngeal dimensions from the normal control group due to the large interindividual variations, except for nasopharyngeal space (PNS-Ad1, $p<$ $0.05)$. More protruded tongue posture was noted in the BSP patients (TT-PTV, $p<0.001)$. In assessments of the dentoskeletal changes after treatment, significant reductions in SNA, SNB, Nper-A, and Nper-B $(p<0.001)$ were observed along with significant reductions in $\mathrm{U} 1-\mathrm{FH}$ and L1-MP $(p<0.001)$. IIA and OB $(p<0.001)$ significantly increased while $0 \mathrm{~J}(p<0.001)$ decreased.

Regarding the pharyngeal and parapharyngeal tissue changes after treatment, SPAS $(p<0.001)$ behind the belly of the soft palate, MAS $(p<0.001)$ behind the soft palate tip, and IAS $(p<0.001)$ at the level of the mandibular plane significantly decreased. In accordance with these findings, the soft palate inclination to the palatal plane (SPI, $p<0.001$ ) increased and tongue position was retruded (TT-PTV, $p<0.001$ ) and lowered (TGH, $p<$ $0.01)$. Nasopharyngeal spaces and vertical airway length showed no significant changes. Notably, however, the pharyngeal airway variable that showed reduction com- pared to the control samples was only MAS $(p<0.01)$, in relation to the retroclined soft palate $(p<0.05)$ and the lowered tongue $(p<0.05)$. Nonetheless, the mean difference in MAS between the posttreatment dimensions in BSP patients $(11.11 \pm 2.85 \mathrm{~mm})$ and the normal dimensions in the controls $(12.64 \pm 1.05 \mathrm{~mm})$ was 1.53 $\mathrm{mm}$, showing little clinical relevance.

\section{Comparisons between hyperdivergent and nonhyperdivergent groups (Tables 2 and 3 )}

Before treatment (Table 2), the hyperdivergent group showed a smaller SPAS $(p<0.01)$ and IAS $(p<0.05)$ than the nonhyperdivergent group. However, the hyperdivergent group showed no significant difference in the pharyngeal dimensions at all levels from the normal controls, while nonhyperdivergent BSP patients exhibited rather larger pharyngeal dimensions than the controls: PNS-Ad1 $(p<0.05)$; SPAS $(p<0.01)$; and IAS $(p<0.05)$.

After treatment (Table 3), changes in the dentoskeletal and facial soft tissue variables showed no significant intergroup differences. For the upper airway variables, both groups showed reductions in SPAS, MAS, IAS in relation to the decrease in TT-PTV and increase in SPI. However, a significant difference in the pharyngeal decrease between the two groups was found only in the MAS ( $p<0.01)$; the hyperdivergent group showed a greater reduction in MAS at the level of the soft palatal tip than the nonhyperdivergent group.

Relationship between posttreatment changes in dentoskeletal and upper airway variables (Tables 4 and 5 )

In the univariable linear regression analysis (Table 4), posttreatment retrusion of the tongue tip (TT-PTV) showed a positive correlation with the retrusion amount of A-point ( $\beta=1.25 ; p<0.01$ ), and posttreatment retroclination of the soft palate (SPI) was correlated with the retrusion amount of B-point $(\beta=-0.49 ; p<0.01)$ and pogonion point $(\beta=-0.58 ; p<0.01)$. On the other hand, posttreatment changes in the pharyngeal airway spaces showed no significant correlation with the retraction amounts of A-point and B-point as well as with the distance of upper and lower incisor retraction. Instead, reductions in SPAS, MAS, IAS were associated with the increases in ANB and FM-MP, respectively. Accordingly, multivariable linear regression analysis was performed using the mandibular plane change as an independent variable adjusted for the changes in the sagittal skeletal relationship (ANB). As a consequence, the posttreatment change in FH-MP showed a negative correlation with the change in MAS $(\beta=-0.42 ; p<0.01)$ and IAS $(\beta=$ $-0.52 ; p<0.01)$. 


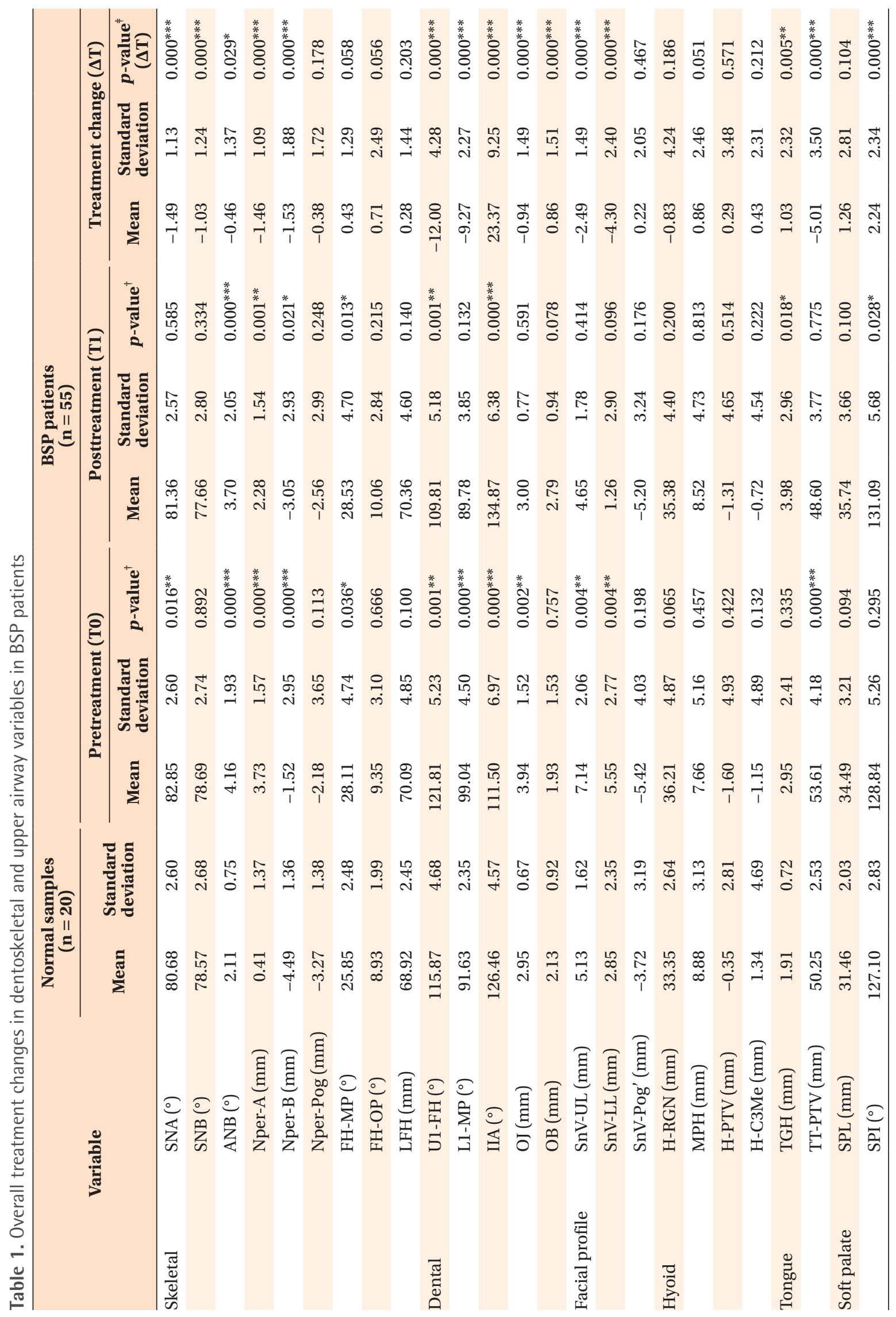




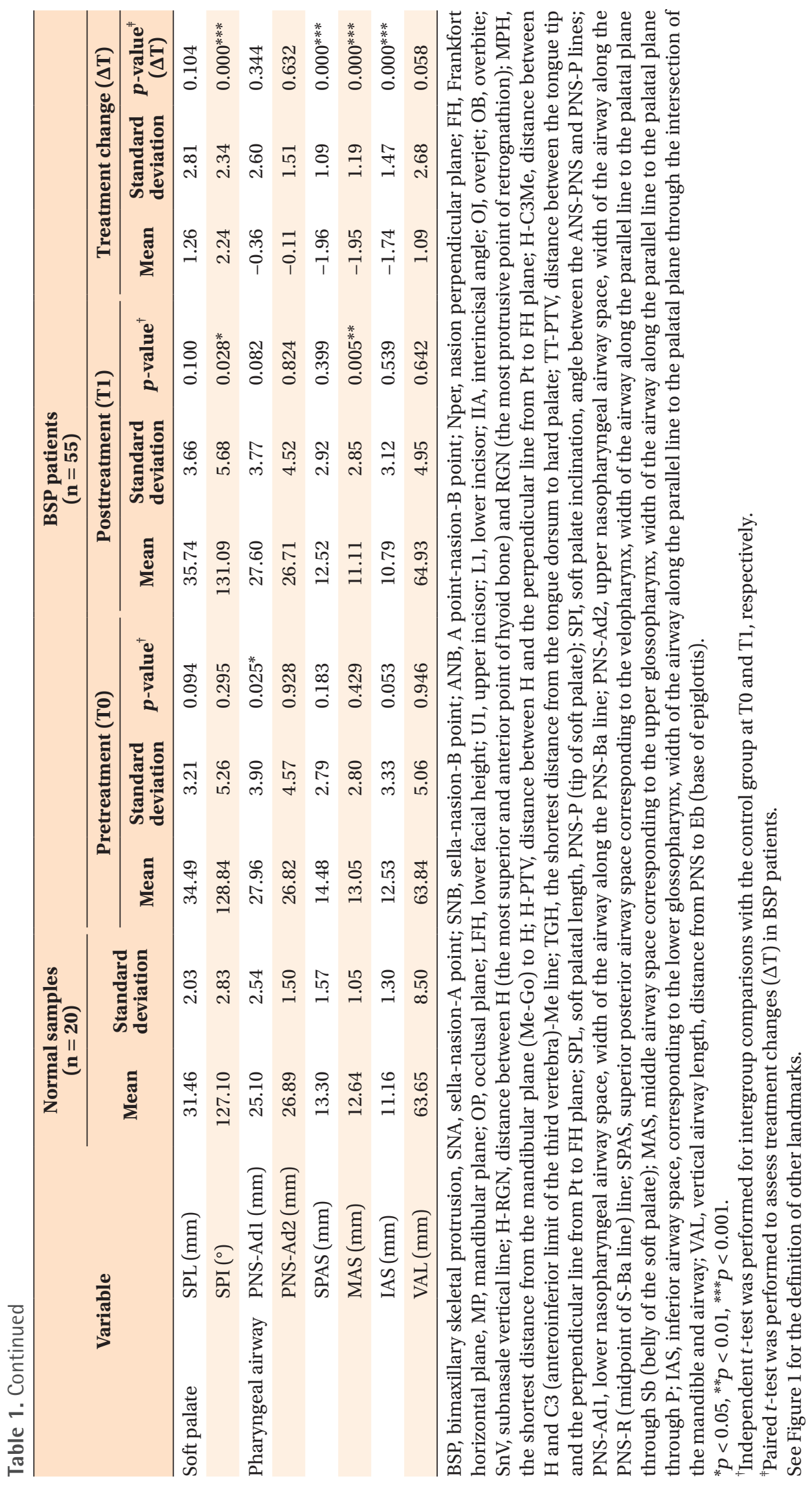




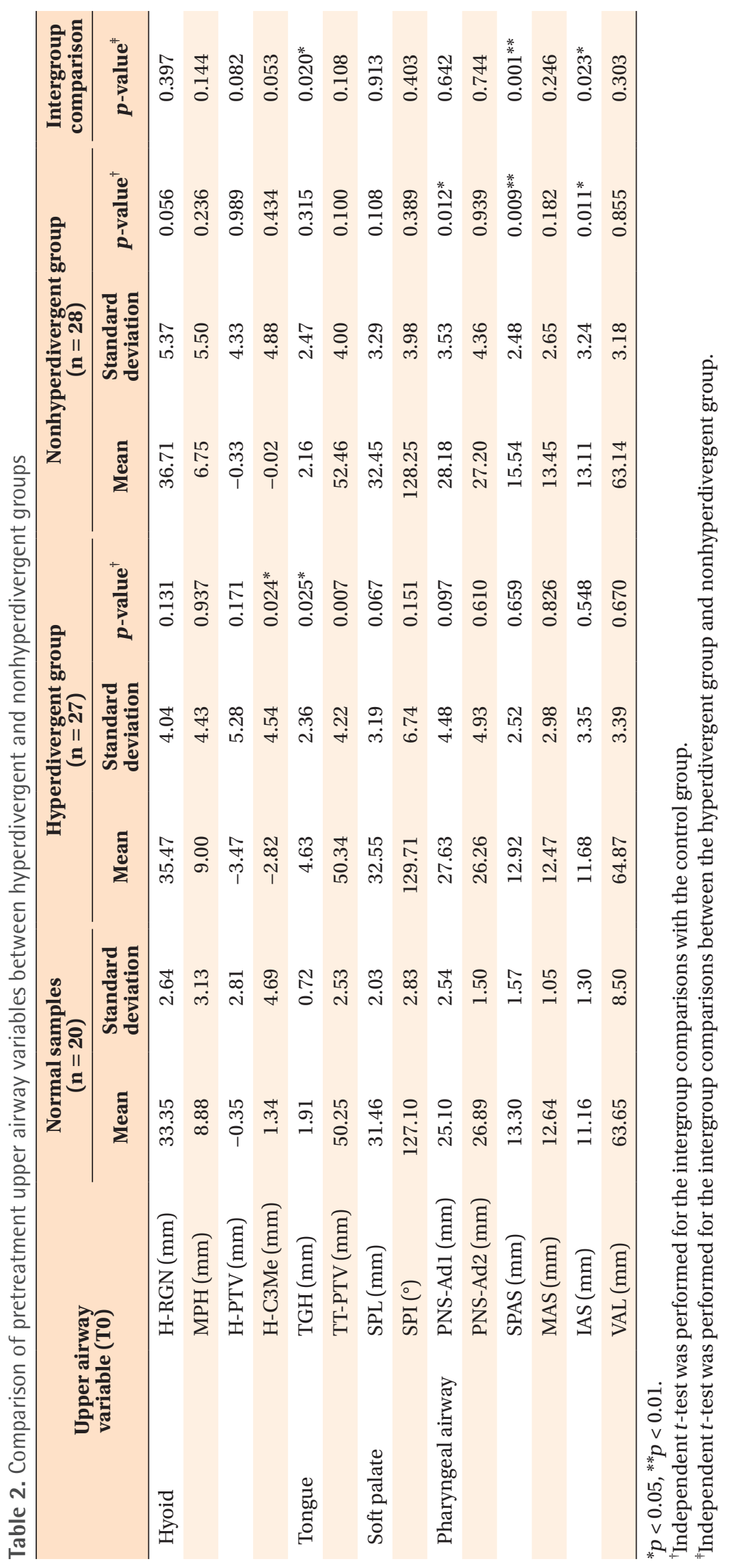




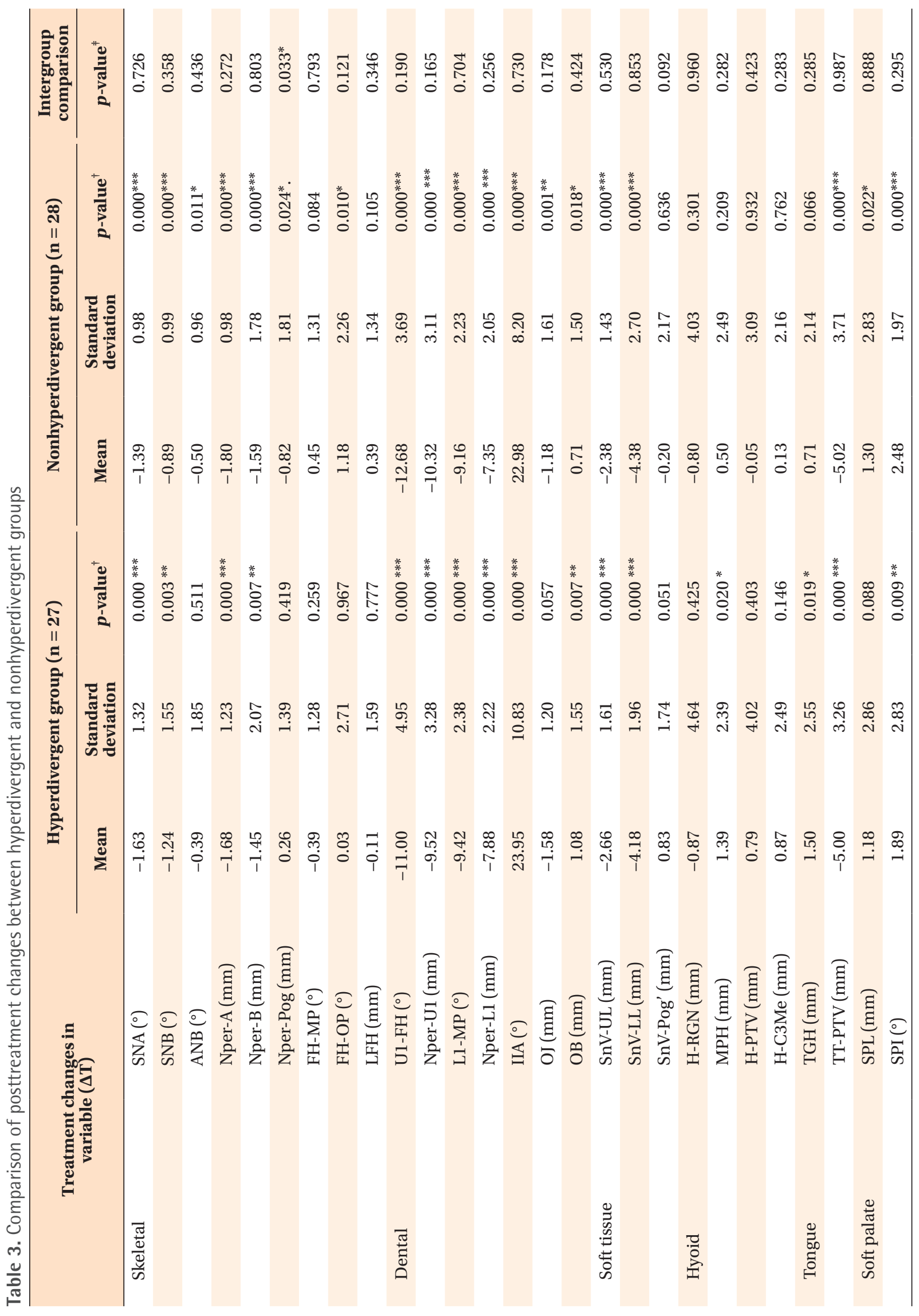




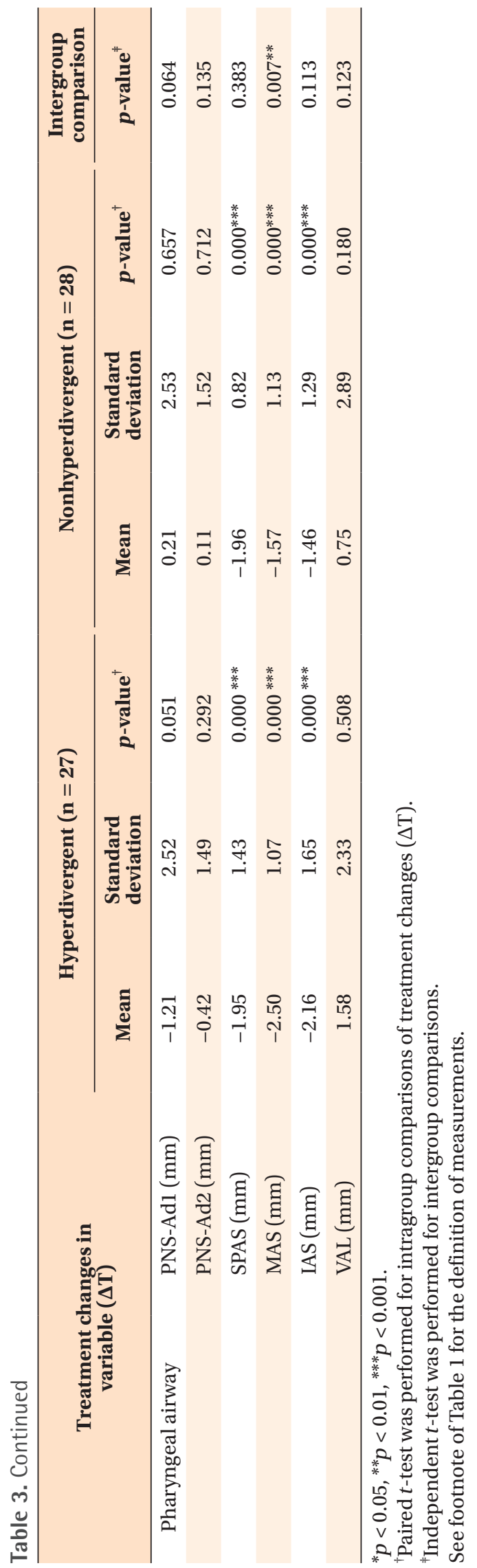

\section{DISCUSSION}

The present study elucidated the influence of premolar extraction treatment with absolute anchorage on the dimensional changes in the upper airway in BSP patients, reflecting the influence of the vertical skeletal pattern. The BSP patients showed significant reductions in velopharyngeal (SPAS) and glossopharyngeal airway spaces (MAS and IAS) after treatment, with no significant changes in nasopharyngeal space (PNS-Ad1 and PNS-Ad2) and vertical airway length (Figure 2). However, the important finding is that the posttreatment pharyngeal narrowing does not indicate an increased risk for functional problems such as snoring or OSA, because the reduced airway dimensions after treatment in the BSP patients mostly corresponded to the normal dimensions in the control samples (Table 1). Uniquely, the posttreatment pharyngeal space at the level of the soft palatal tip (MAS) was smaller than the normal MAS value by $1.53 \mathrm{~mm}$, which may not be clinically relevant. When the pharyngeal dimensional changes were compared between the two groups, the hyperdivergent BSP group showed a greater reduction in the MAS than the nonhyperdivergent BSP group by $0.93 \mathrm{~mm}$ (Table 3). In addition, the posttreatment increase in the mandibular plane angle might affect the reductions of MAS and IAS in BSP patients (Table 5). This study demonstrates that the pharyngeal dimensions of BSP patients tended to be resistant to the anteroposterior changes with maximum anterior retraction, while the glossopharyngeal space might become susceptible to the vertical increase after extraction treatment.

Previous studies investigated the pharyngeal airway changes after extraction treatment mostly in BMP patients, with no special consideration of double jaw protrusion. Although it has been difficult to draw a qualified conclusion due to the limited number of studies and heterogeneity across studies, a systematic review ${ }^{21}$ summarized that premolar extraction followed by a large retraction of the anterior teeth might lead to upper airway constriction in BMP patients. However, previous findings have never been supported by comparisons with normal pharyngeal dimensions obtained from control samples with normal skeletal and dental occlusion. It is clinically important to understand what the posttreatment upper airway dimension implies in relation to the dentoskeletal changes considering the normal range of airway patency, rather than limiting the interpretation just on the absolute amount of airway change. The present study could show that the retruded tongue position and reduced velopharyngeal spaces after treatment in BSP patients fell within normal ranges as in the control samples, despite the maximum retraction of the upper and lower incisors and anterior alveolus. ${ }^{15,17,22}$ 


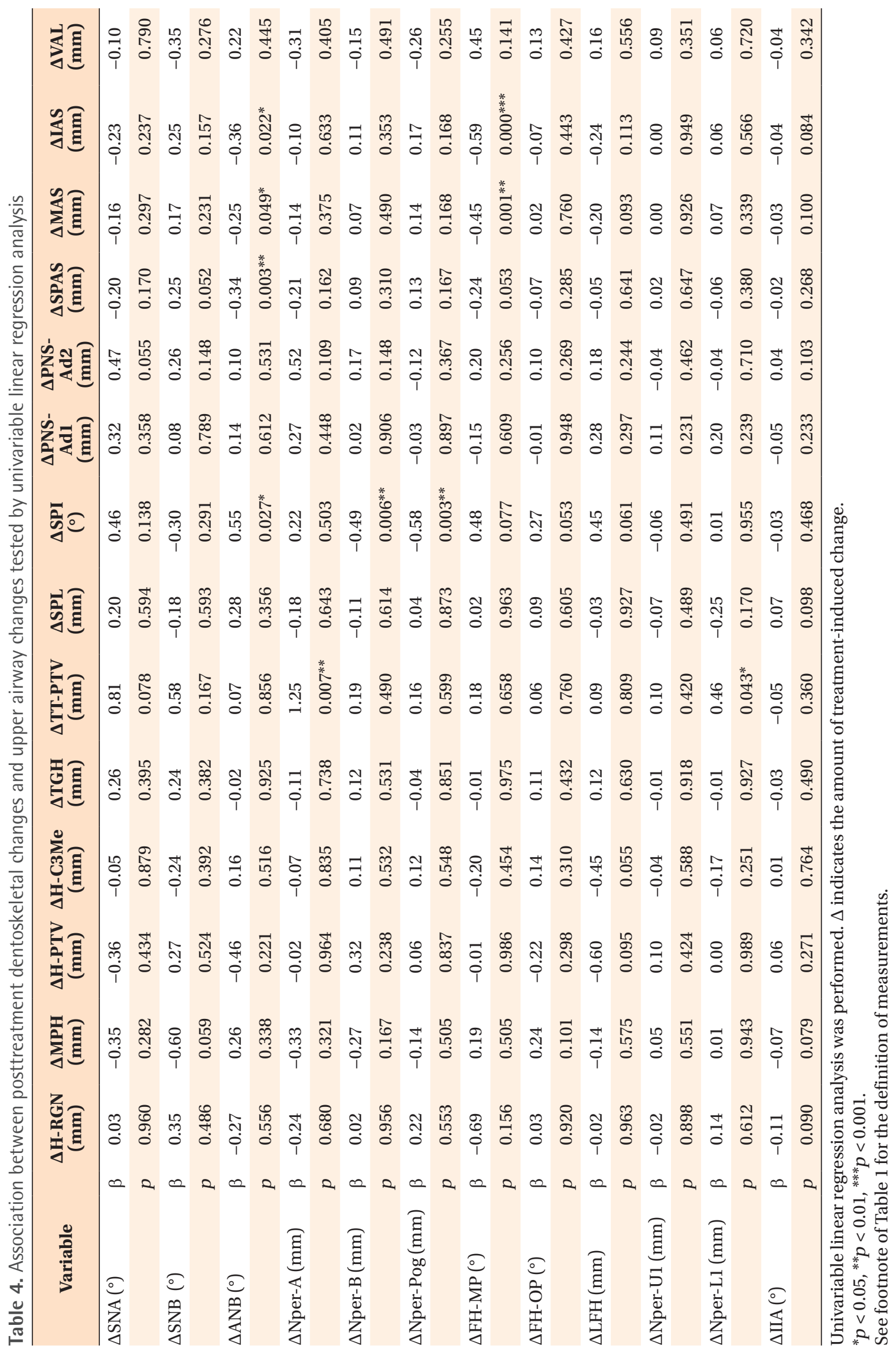


Table 5. Posttreatment changes in upper airway variables according to the change in mandibular plane steepness, tested by multivariable linear regression analysis adjusted for sagittal skeletal classification

\begin{tabular}{|c|c|c|c|c|}
\hline \multirow{3}{*}{$\begin{array}{l}\text { Dependent } \\
\text { variables }\end{array}$} & \multicolumn{4}{|c|}{ Independent variable } \\
\hline & \multicolumn{4}{|c|}{$\Delta$ FH-MP $\left(^{\circ}\right)$} \\
\hline & $\beta$-estimate & & CI & $p$-value \\
\hline$\Delta \mathrm{H}-\mathrm{RGN}(\mathrm{mm})$ & -0.70 & -1.80 & 0.39 & 0.202 \\
\hline$\Delta \mathrm{MPH}(\mathrm{mm})$ & 0.09 & -0.56 & 0.73 & 0.791 \\
\hline$\Delta \mathrm{H}-\mathrm{PTV}(\mathrm{mm})$ & 0.27 & -0.63 & 1.17 & 0.553 \\
\hline$\Delta \mathrm{H}-\mathrm{C} 3 \mathrm{Me}(\mathrm{mm})$ & -0.35 & -0.95 & 0.25 & 0.246 \\
\hline$\Delta \mathrm{TGH}(\mathrm{mm})$ & 0.00 & -0.61 & 0.62 & 0.990 \\
\hline$\Delta \mathrm{TT}-\mathrm{PTV}(\mathrm{mm})$ & 0.18 & -0.74 & 1.11 & 0.689 \\
\hline$\Delta \mathrm{SPL}(\mathrm{mm})$ & -0.15 & -0.88 & 0.58 & 0.683 \\
\hline$\Delta \mathrm{SPI}\left({ }^{\circ}\right)$ & 0.27 & -0.32 & 0.85 & 0.363 \\
\hline$\Delta$ PNS-Adl (mm) & -0.28 & -0.96 & 0.40 & 0.411 \\
\hline$\triangle \mathrm{PNS}-\mathrm{Ad} 2(\mathrm{~mm})$ & 0.19 & -0.21 & 0.58 & 0.344 \\
\hline$\Delta \mathrm{SPAS}(\mathrm{mm})$ & -0.10 & -0.36 & 0.16 & 0.443 \\
\hline$\Delta$ MAS (mm) & -0.42 & -0.69 & -0.14 & $0.004^{* *}$ \\
\hline$\Delta \mathrm{IAS}(\mathrm{mm})$ & -0.52 & -0.85 & -0.19 & $0.003^{* *}$ \\
\hline$\triangle \mathrm{VAL}(\mathrm{mm})$ & 0.44 & -0.26 & 1.13 & 0.211 \\
\hline
\end{tabular}

Multivariable linear regression analysis was performed using the mandibular plane angle (FH-MP) change as an independent variable adjusted for the ANB angle. $\Delta$ indicates the amount of treatment change.

CI, confidence interval.

** $p<0.01$.

See footnote of Table 1 for the definition of measurements.

Previous studies have attempted to identify dentoskeletal predictive factors for the pharyngeal airway changes after extraction treatment, mostly in the horizontal direction. Wang et al. ${ }^{10}$ suggested a correlation between the retraction distance of the lower incisors and reductions in velopharyngeal and glossopharyngeal spaces in BMP patients in a cephalometric study, but their measurements focused on dental parameters with no definite description of the maxillary and mandibular jaw positions. Chen et al. ${ }^{9}$ reported a correlation between the retraction distance of the upper incisors and hypopharyngeal constriction in a cone-beam computed tomographic (CBCT) study, but their samples were irrelevant to the BSP population. Recently, Zheng et al..$^{23}$ found correlations among pressure, airflow, and pharyngeal cross-sectional reductions in BMP patients by performing a computational fluid dynamics study, but they did not address dentoskeletal risk factors. Interestingly, the present study suggested that the vertical skeletal change with clockwise mandibular rotation might be a risk factor for glossopharyngeal narrowing in BSP patients, as supported by multivariable linear regression analysis using the FH-MP as an independent variable adjusted for the sagittal skeletal relationship. The clinical significance of this finding is that it would be more important to avoid the increasement of the vertical dimension than to compromise the amount of incisor retraction in the treatment of BSP patients with respiratory concerns, in order to achieve both esthetic and functional respiratory treatment goals.

Regarding the mechanism underlying the pharyngeal narrowing after maximum retraction, the backward displacement of the tongue base, soft palate, and hyoid bone have been suspected. ${ }^{15,21}$ Backward displacement of the tongue along with a decrease in the oral cavity pushes the soft palate backward via the communicating action of palatoglossus muscles, leading to velopharyngeal and glossopharyngeal airway reductions in the horizontal direction. ${ }^{19,22}$ The impact of hyoid positional changes on the upper airway has been a topic of debate among the studies since it depended on the presence or absence of compensatory positional adaptation to prevent encroachment of the tongue into the pharynx. ${ }^{7,24}$ In the present study, posttreatment retrusion of the tongue tip (TT-PTV) showed a positive correlation with the retrusion amount of A-point, and posttreatment retroclination of the soft palate (SPI) was correlated with the retrusion amount of B-point and pogonion point. On the other hand, posttreatment changes in the pharyngeal airway spaces and hyoid bone showed no significant correlation with the amount of dentoalveolar retraction. Notably, the positional changes in parapharyngeal soft tissues showed too little reliability or reproducibility in lateral cephalography to have clinical significance for the posttreatment pharyngeal changes. Our understanding of the physiologic mechanism of functional adaptation and restoration of the tissues needs to be strengthened.

It is still unclear whether the pharyngeal narrowing with parapharyngeal tissue displacement after extraction treatment might bring about stable functional adaptation in the long term. Nevertheless, in consideration of various phenotypic causes of OSA, including the anatomical and nonanatomical factors, ${ }^{25}$ it should be noted that even BSP patients might have nonanatomical OSA risks irrelevant to the craniofacial condition. In this context, upper airway dimensions and respiratory or sleep functions need to be maintained under control by orthodontists, especially when maximum anterior retraction treatment is planned for the hyperdivergent BSP patients with pharyngeal constriction and respiratory symptoms.

The present study included the following fundamental limitations: (1) morphological image analysis of pharyngeal airway taken in the awake state and upright position; (2) difficulty in standardization of cephalometry to 
represent consistent parapharyngeal soft tissue positions; (3) no inclusion of three-dimensional analysis of the skeletal pattern and pharyngeal volume; (4) the absence of long-term treatment changes matched with followup functional investigations. Although the pharyngeal changes in the transverse dimension could not be reflected in the present analysis, our study had the significance of attempting pharyngeal airway prediction in relation to the sagittal and vertical dentoskeletal treatment-induced changes by using the routinely employed screening tool of lateral cephalometry. It is still clinically possible to see the dentoskeletal pattern, pharyngeal morphology and dimension, and parapharyngeal soft tissues at a glance in planning orthodontic treatment. Further studies are ongoing to evaluate the relationship between altered upper airway dimension after orthodontic treatment and the resultant effects on respiratory or sleep function, based on a three-dimensional CBCT analysis related to polysomnographic studies.

\section{CONCLUSION}

1. The BSP patients showed significant reductions in the velopharyngeal and glossopharyngeal airway spaces after extraction treatment; however the reduced posttreatment dimensions corresponded to the normal dimensions in the control samples.

2. The glossopharyngeal space might be susceptible after treatment in the hyperdivergent BSP group, especially when the mandibular plane angle is increased during treatment.

3. Control of the vertical dimensions during maximum anterior retraction would be important to achieve both esthetic and respiratory functional improvements in BSP patients.

\section{CONFLICTS OF INTEREST}

No potential conflict of interest relevant to this article was reported.

\section{REFERENCES}

1. Kim TK, Ryu YK. Lip profile changes after orthodontic tooth movement in female adult with bimaxillary protrusion. Korean J Orthod 1994;24:135-48.

2. Chu YM, Po-Hsun Chen R, Morris DE, Wen-Ching Ko E, Chen YR. Surgical approach to the patient with bimaxillary protrusion. Clin Plast Surg 2007;34:53546.

3. Bills DA, Handelman CS, BeGole EA. Bimaxillary dentoalveolar protrusion: traits and orthodontic correction. Angle Orthod 2005;75:333-9.

4. Leonardi R, Annunziata A, Licciardello V, Barbato
E. Soft tissue changes following the extraction of premolars in nongrowing patients with bimaxillary protrusion. A systematic review. Angle Orthod 2010;80:211-6.

5. Uesugi S, Imamura T, Kokai S, Ono T. Cone-beam computed tomography-based diagnosis and treatment simulation for a patient with a protrusive profile and a gummy smile. Korean J Orthod 2018; 48:189-99.

6. Sharma K, Shrivastav S, Sharma N, Hotwani K, Murrell MD. Effects of first premolar extraction on airway dimensions in young adolescents: a retrospective cephalometric appraisal. Contemp Clin Dent 2014;5:190-4.

7. Valiathan M, El H, Hans MG, Palomo MJ. Effects of extraction versus non-extraction treatment on oropharyngeal airway volume. Angle Orthod 2010; 80:1068-74.

8. Jones AG, Bhatia S. A study of nasal respiratory resistance and craniofacial dimensions in white and West Indian black children. Am J Orthod Dentofacial Orthop 1994;106:34-9.

9. Chen Y, Hong L, Wang CL, Zhang SJ, Cao C, Wei $F$, et al. Effect of large incisor retraction on upper airway morphology in adult bimaxillary protrusion patients. Angle Orthod 2012;82:964-70.

10. Wang Q, Jia P, Anderson NK, Wang L, Lin J. Changes of pharyngeal airway size and hyoid bone position following orthodontic treatment of Class 1 bimaxillary protrusion. Angle Orthod 2012;82:11521.

11. Al Maaitah E, El Said N, Abu Alhaija ES. First premolar extraction effects on upper airway dimension in bimaxillary proclination patients. Angle Orthod 2012;82:853-9.

12. Zheng ZH, Yamaguchi T, Kurihara A, Li HF, Maki K. Three-dimensional evaluation of upper airway in patients with different anteroposterior skeletal patterns. Orthod Craniofac Res 2014;17:38-48.

13. Baik UB, Suzuki M, Ikeda K, Sugawara J, Mitani H. Relationship between cephalometric characteristics and obstructive sites in obstructive sleep apnea syndrome. Angle Orthod 2002;72:124-34.

14. Joseph AA, Elbaum J, Cisneros GJ, Eisig SB. A cephalometric comparative study of the soft tissue airway dimensions in persons with hyperdivergent and normodivergent facial patterns. J Oral Maxillofac Surg 1998;56:135-9; discussion 139-40.

15. Ucar Fl, Uysal T. Orofacial airway dimensions in subjects with Class 1 malocclusion and different growth patterns. Angle Orthod 2011;81:460-8.

16. Behrents RG, Shelgikar AV, Conley RS, Flores-Mir C, Hans M, Levine M, et al. Obstructive sleep apnea and orthodontics: an American Association of Or- 
thodontists White Paper. Am J Orthod Dentofacial Orthop 2019;156:13-28.e1.

17. Marşan G, Cura N, Emekli U. Changes in pharyngeal (airway) morphology in Class 111 Turkish female patients after mandibular setback surgery. J Craniomaxillofac Surg 2008;36:341-5.

18. Keum BT, Choi SH, Choi YJ, Baik HS, Lee KJ. Effects of bodily retraction of mandibular incisors versus mandibular setback surgery on pharyngeal airway space: a comparative study. Korean J Orthod 2017;47:344-52.

19. Germec-Cakan D, Taner T, Akan S. Uvulo-glossopharyngeal dimensions in non-extraction, extraction with minimum anchorage, and extraction with maximum anchorage. Eur J Orthod 2011;33:515-20.

20. Iwasaki T, Saitoh I, Takemoto Y, Inada E, Kakuno E, Kanomi R, et al. Tongue posture improvement and pharyngeal airway enlargement as secondary effects of rapid maxillary expansion: a cone-beam computed tomography study. Am J Orthod Dentofacial Orthop 2013;143:235-45.

21. Hu Z, Yin X, Liao J, Zhou C, Yang Z, Zou S. The ef- fect of teeth extraction for orthodontic treatment on the upper airway: a systematic review. Sleep Breath 2015;19:441-51.

22. Jena AK, Singh SP, Utreja AK. Sagittal mandibular development effects on the dimensions of the awake pharyngeal airway passage. Angle Orthod 2010;80:1061-7.

23. Zheng Z, Liu H, Xu Q, Wu W, Du L, Chen H, et al. Computational fluid dynamics simulation of the upper airway response to large incisor retraction in adult class 1 bimaxillary protrusion patients. Sci Rep 2017;7:45706.

24. Eggensperger N, Smolka W, lizuka T. Long-term changes of hyoid bone position and pharyngeal airway size following mandibular setback by sagittal split ramus osteotomy. J Craniomaxillofac Surg 2005;33:111-7.

25. Zinchuk AV, Gentry MJ, Concato J, Yaggi HK. Phenotypes in obstructive sleep apnea: a definition, examples and evolution of approaches. Sleep Med Rev 2017;35:113-23. 